\title{
BROWNIAN DYNAMICS SIMULATION OF CHAIN FORMATION IN ELEC- TRORHEOLOGICAL FLUIDS
}

\author{
DÁvID FERTIG*1 ${ }^{*}$ DEZSŐ BOdA ${ }^{1}$, AND ISTVÁN SZALA2 ${ }^{2,3}$ \\ ${ }^{1}$ Department of Physical Chemistry, University of Pannonia, Egyetem u. 10, Veszprém, 8200, HUNGARY \\ ${ }^{2}$ Institute of Physics and Mechatronics, University of Pannonia, Egyetem u. 10, Veszprém, 8200, HUNGARY \\ ${ }^{3}$ Institute of Mechatronics Engineering and Research, University of Pannonia, Gasparich Márk u. 18/A, \\ Zalaegerszeg, 8900, HUNGARY
}

\begin{abstract}
Brownian dynamics (BD) simulations based on a novel Langevin integrator algorithm are used to simulate the dynamics of chain formation in electrorheological (ER) fluids that are non-conducting solid particles suspended in a liquid that has a dielectric constant different from that of the ER particles. An external electric field induces polarization charge distributions on the spheres' surfaces that can be modeled as point dipoles in the centers of the spheres. The interaction of these aligned dipoles leads to formation of chains and other aggregates in the ER fluid. In this work, we introduce our methodology and report results for various quantities characterizing the structure of the ER system as obtained with BD simulations. These quantities include the potential energy, diffusion constant, average chain length, chain length distributions, and pair correlation functions. Their behavior as a function of time is presented as the electric field is switched on. The properties of the ER fluid change considerably making this system a potential basic material of many applications.
\end{abstract}

Keywords: electrorheological fluids, chain formation, Brownian dynamics

\section{Introduction}

Electrorheological (ER) fluids are [1] suspensions of fine non-conducting solid particles in an electrically insulating liquid. If the particles, imagined as closely spherical, have a dielectric constant that is different from that of the solvent, the arising dielectric boundaries respond to an applied electric field. This dielectric response is the polarization of the spheres resulting in a polarization charge distribution whose dominant component in the multipole expansion is the dipole moment.

The interactions of these dipoles then lead to a structural change in the ER fluid known as the ER response. This structural change is basically a formation of chains and other forms of clusters as the polarized spheres are linked together into head-to-tail positions. This structural phase transition is reversible and relatively fast.

This structural change results in a dramatic change in the physical properties of the ER fluid of which the most important is viscosity. This externally controllable, fast and reversible change in viscosity makes ER fluids a kind of a smart material, a central component of devices, such as brakes, clutches, dampers, and valves [2,3]. Such devices have crucial importance in the industry of various fields.

\footnotetext{
*Correspondence: fertig.david92@gmail.com
}

The continuously shrinking size of devices resulted in the development of nanotechnology. Understanding the molecular mechanisms behind the workings of nanodevices is especially important because better understanding of microscopic mechanisms can lead to novel designs.

ER devices are also based on microscopic mechanisms leading to an emergent macroscopic pattern. No wonder that many modeling studies [4-22] aimed at investigating the microscopic processes behind chain formation and corresponding changes in measurable physical properties.

The properties of the ER fluid in the absence of an applied electric field have been investigated by Heyes and Melrose [23]. This means the investigation of the core potential that is either the Lennard-Jones (LJ) fluid or its cut-and-shifted version that is a purely repulsive potential. It has been demonstrated that the repulsive version reproduces experimental behavior better [4].

Cluster formation has been investigated via cluster size distribution $[4,9,11,12,20,22]$, order parameters $[12-15,19]$, mean square displacement and diffusion constant $[4,6,12]$, pair distribution functions $[6,12]$, and relaxation times $[5,11,12,21]$. In particular, Cao et al. [21] identified relaxation times corresponding to various subprocesses such as initial aggregation, chain formation, and column formation. Identifying these subprocesses is 


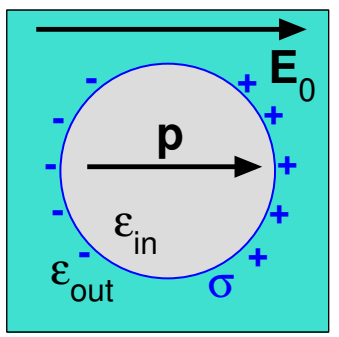

Figure 1: Sketch of an ER particle in an external electric field, $\mathbf{E}_{0}$. The dielectric constant inside the sphere is $\epsilon_{\text {in }}$, while outside the sphere is $\epsilon_{\text {out }}$. The surface charge distribution, $\sigma(\mathbf{r})$, induced on the dielectric boundary (Eq. 1) can be approximated by a point dipole, $\mathbf{p}$, in the center of the sphere (Eq. 2).

also our long-term goal. It is also our intention to simulate the ER system in the presence of shear as several authors did $[5,6,10,15,21]$. These authors investigated shear stress, various terms of viscosity, oscillatory strain, and dependence on strain rate.

In this paper, we do not apply stress, because our main interest is to study the dynamics of the formation of chains with a newly developed simulation package based on a novel Langevin integrator [24-26] as opposed to most studies from the 1990s that used the overdamped limit. We intend to test the program on the ER fluid in the absence and presence of an applied electric field and to follow the dynamics of chain formation when the field is switched on. We characterize this dynamics by plotting energy, mean square displacement, diffusion constant, average chain length, chain length distributions, and radial distribution functions as functions of time.

We use reduced units in this study (see Section 4) that are closely related to various parameters used in the literature. These parameters characterize the relations of various effects in the ER fluid. These effects are the polarization (dipole-dipole), thermal, and viscous forces.

The relation of the polarization and thermal forces is often denoted by $\lambda$ and it practically corresponds to the square of the reduced dipole moment used in this study. It expresses the relation of the ordering effect of electrostatic forces and the disordering effect of thermal motion. The relation of the viscous force to the electrostatic force is called the Mason number (Ma). Many authors plot the characteristic physical quantities as functions of the Mason number $[5,10,15]$. The relation of the viscous and the electrostatic forces is called the Péclet number.

\section{Model: the polarizable dielectric sphere}

We model the ER fluid as dielectric spheres of dielectric constant $\epsilon_{\text {in }}$ inside the sphere immersed in a fluid of dielectric constant $\epsilon_{\text {out }}$ (Fig. 1). The radius of the spheres is $R$, while their diameter is $d=2 R$. When a constant electric field, $\mathbf{E}_{0}$ is applied to this system (in the $z$ direction), the dielectric boundary on the sphere's surface becomes

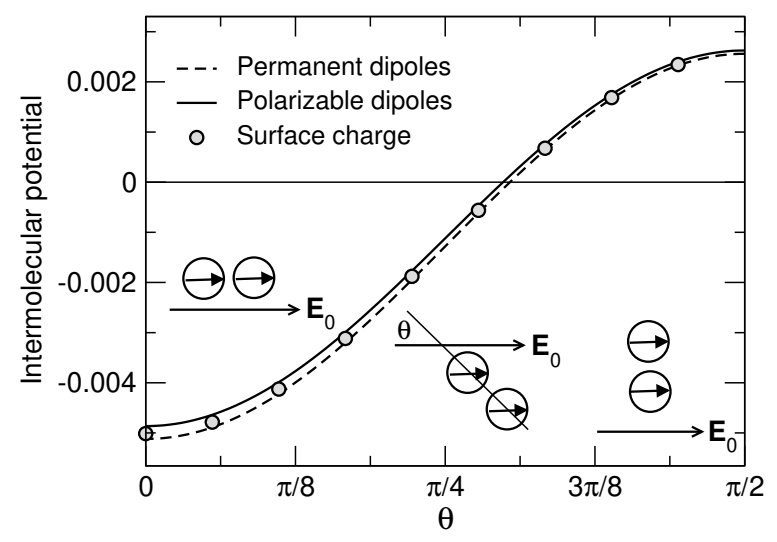

Figure 2: Interaction potential (arbitrary unit) between two dipoles at $r=1.25 d$ distance from each other at different mutual positions characterized by angle $\theta$ that is the angle between $\mathbf{E}_{0}$ and $\mathbf{r}_{i j}$. The potential is computed from the interaction of the charge distributions in Eq. 1 using the ICC method (symbols), from the interactions of the permanent point dipoles induced only by $\mathbf{E}_{0}$ (Eq. 2) (dashed line), and from the interaction of the polarizable dipoles when the sphere can be polarized by the electric field of other dipoles too (solid line).

polarized. The polarization charge density is

$$
\sigma(\theta)=3 \epsilon_{0}\left(\frac{\epsilon_{\text {in }}-\epsilon_{\text {out }}}{\epsilon_{\text {in }}+2 \epsilon_{\text {out }}}\right) E_{0} \cos \theta,
$$

where $E_{0}=\left|\mathbf{E}_{0}\right|, \theta$ is the angle between the point of on the surface and the $z$-axis, and $\epsilon_{0}$ is the permittivity of vacuum. As it was discussed in our previous publication [30], the effect of this surface charge distribution can be approximated with an ideal point dipole placed in the center of the sphere computed as [31]

$$
\mathbf{p}=4 \pi \epsilon_{0}\left(\frac{\epsilon_{\text {in }}-\epsilon_{\text {out }}}{\epsilon_{\text {in }}+2 \epsilon_{\text {out }}}\right) R^{3} \mathbf{E}_{0} .
$$

In that paper, we showed that the point dipole model is a good approximation to the exact solution obtained from the polarization charge using the Induced Charge Computation method [32]. The agreement is better if the spheres are assumed to be polarizable by the electric fields of all the other particles, but even if it is assumed that an ER particle is polarized only by $\mathbf{E}_{0}$, the agreement is reasonable (Fig 2). The latter assumption means that the ER particles carry only the permanent dipoles of Eq. 2 that always point into the $z$-direction.

We further assume that the characteristic time of the rearrangement of the surface charge as the particles move is much smaller than the characteristic time of the rotation of the particles. This means that the $\mathbf{p}$ dipole always points into the $z$ direction even if the sphere rotates, because the induced charges (that chiefly correspond to polarization of solvent molecules around the sphere) always have enough time to rearrange themselves according to the applied field, $\mathbf{E}_{0}$.

The potential produced by a dipole $\mathbf{p}_{j}$ (that is at $\mathbf{r}_{j}$ ) 
at the position $\mathbf{r}_{i}$ of another dipole $\mathbf{p}_{i}$ is

$$
\Phi_{j}\left(\mathbf{r}_{i}\right)=\frac{1}{4 \pi \epsilon_{0}} \frac{\mathbf{p}_{j} \cdot \mathbf{r}_{i j}}{r_{i j}^{3}},
$$

where $\mathbf{r}_{i j}=\mathbf{r}_{i}-\mathbf{r}_{j}$ and $r_{i j}=\left|\mathbf{r}_{i j}\right|$. The electric field is

$$
\mathbf{E}_{j}\left(\mathbf{r}_{i}\right)=\frac{1}{4 \pi \epsilon_{0}} \frac{3 \mathbf{n}_{i j}\left(\mathbf{n}_{i j} \cdot \mathbf{p}_{j}\right)-\mathbf{p}_{j}}{r_{i j}^{3}},
$$

where $\mathbf{n}_{i j}=\mathbf{r}_{i j} / r_{i j}$. The interaction potential between the two dipoles is

$$
\begin{array}{r}
u_{i j}^{\mathrm{DD}}\left(\mathbf{r}_{i j}, \mathbf{p}_{i}, \mathbf{p}_{j}\right)=-\mathbf{p}_{i} \cdot \mathbf{E}_{j}\left(\mathbf{r}_{i}\right)= \\
=-\frac{1}{4 \pi \epsilon_{0}} \frac{3\left(\mathbf{n}_{i j} \cdot \mathbf{p}_{i}\right)\left(\mathbf{n}_{i j} \cdot \mathbf{p}_{j}\right)-\mathbf{p}_{i} \cdot \mathbf{p}_{j}}{r_{i j}^{3}},
\end{array}
$$

while the force exerted on dipole $\mathbf{p}_{i}$ by dipole $\mathbf{p}_{j}$ is

$$
\begin{array}{r}
\mathbf{f}_{i j}^{\mathrm{DD}}\left(\mathbf{r}_{i j}, \mathbf{p}_{i}, \mathbf{p}_{j}\right)=-\left(\mathbf{p}_{i} \cdot \nabla_{i}\right) \mathbf{E}_{j}\left(\mathbf{r}_{i}\right)= \\
=\frac{1}{4 \pi \epsilon_{0}} \frac{1}{r_{i j}^{4}}\left\{3 \left[\mathbf{p}_{i}\left(\mathbf{n}_{i j} \cdot \mathbf{p}_{j}\right)+\mathbf{p}_{j}\left(\mathbf{n}_{i j} \cdot \mathbf{p}_{i}\right)+\right.\right. \\
\left.\left.+\mathbf{n}_{i j}\left(\mathbf{p}_{i} \cdot \mathbf{p}_{j}\right)\right]-15 \mathbf{n}_{i j}\left(\mathbf{n}_{i j} \cdot \mathbf{p}_{i}\right)\left(\mathbf{n}_{i j} \cdot \mathbf{p}_{j}\right)\right\} .
\end{array}
$$

Note that the forms of these equations are simplified when all the dipoles of magnitude $p$ are aligned in the $z$ direction:

$$
u_{i j}^{\mathrm{DD}}\left(r_{i j}, \theta\right)=-\frac{p^{2}}{4 \pi \epsilon_{0}} \frac{3 \cos ^{2} \theta-1}{r_{i j}^{3}},
$$

and

$$
\mathbf{f}^{\mathrm{DD}}\left(r_{i j}, \theta\right)=\frac{3 p^{2}}{4 \pi \epsilon_{0}} \frac{(2 \cos \theta) \mathbf{k}+\left(1-5 \cos ^{2} \theta\right) \mathbf{n}_{i j}}{r_{i j}^{4}},
$$

where $\mathbf{k}$ is the unit vector in the direction of the $z$-axis and $\theta$ is the angle between $\mathbf{k}$ and $\mathbf{n}_{i j}$. There is also a torque acting on the dipole, but because the characteristic time of polarization charge formation is much smaller than the characteristic time of the rotation of the sphere, the rearrangement of surface charges is considered instantaneous without inertia. The torque, therefore, has been neglected.

The full interaction potential between two ER particles consists of this dipole-dipole (DD) term and a shortrange core potential that defines the finite size of the particles:

$$
u_{i j}=u_{i j}^{\mathrm{DD}}+u_{i j}^{\mathrm{WCA}} .
$$

For the core potential, we use the cut $\&$ shifted LJ potential also known as the Weeks-Chandler-Anderson (WCA) potential that is

$$
u_{i j}^{\mathrm{WCA}}\left(r_{i j}\right)=\left\{\begin{array}{lll}
u_{i j}^{\mathrm{LJ}}\left(r_{i j}\right)+u_{i j}^{\mathrm{LJ}}\left(r_{\mathrm{c}}\right) & \text { if } & r_{i j}<r_{\mathrm{c}} \\
0 & \text { if } & r_{i j}>r_{\mathrm{c}}
\end{array},\right.
$$

where

$$
u_{i j}^{\mathrm{LJ}}\left(r_{i j}\right)=4 \varepsilon^{\mathrm{LJ}}\left[\left(\frac{d}{r_{i j}}\right)^{12}-\left(\frac{d}{r_{i j}}\right)^{6}\right]
$$

is the $\mathrm{LJ}$ potential. The WCA force is

$$
\mathbf{f}_{i j}^{\mathrm{WCA}}\left(\mathbf{r}_{i j}\right)=\left\{\begin{array}{ll}
\mathbf{f}_{i j}^{\mathrm{LJ}}\left(\mathbf{r}_{i j}\right) & \text { if } r_{i j}<r_{\mathrm{c}} \\
0 & \text { if } \quad r_{i j}>r_{\mathrm{c}}
\end{array},\right.
$$

where

$$
\mathbf{f}_{i j}^{\mathrm{LJ}}\left(\mathbf{r}_{i j}\right)=24 \varepsilon^{\mathrm{LJ}}\left[2\left(\frac{d}{r_{i j}}\right)^{12}-\left(\frac{d}{r_{i j}}\right)^{6}\right] \frac{\mathbf{r}_{i j}}{r_{i j}^{2}}
$$

is the $\mathrm{LJ}$ force. In these equations the cutoff distance is $r_{\mathrm{c}}=2^{1 / 6} d$ that is at the minimum of the $\mathrm{LJ}$ potential, so this potential is a smooth repulsive core potential used widely in dynamical simulations of large spherical particles.

\section{Method: Brownian Dynamics simulation}

When it comes to simulating the trajectories of particles in the phase space interacting with each other via a systematic force, $\mathbf{f}_{i j}$ (like those given in Eqs. 6 and 12), we use Newton's equation of motion in an MD simulation. In this case, the particles move in vacuum and the only forces that we take into account are those exerted by the particles themselves (plus, possibly, external forces).

When it comes to simulating the trajectories of particles immersed in a solvent, we use Langevin's equations of motion [33]

$$
m \frac{d \mathbf{v}_{i}(t)}{d t}=\mathbf{F}_{i}\left(\mathbf{r}_{i}(t)\right)-m \gamma \mathbf{v}_{i}(t)+\mathbf{R}_{i}(t)
$$

where $\mathbf{r}_{i}, \mathbf{v}_{i}, m$, and $\gamma$ are the position, the velocity, the mass, and the friction coefficient of particle $i$, respectively. The mass and the friction coefficient are assumed to be the same for every particle, but, in general, they can depend on $i$.

The force has three components. In addition to the systematic force, $\mathbf{F}_{i}\left(\mathbf{r}_{i}(t)\right)=\sum_{j \neq i} \mathbf{f}_{i j}$, there are the frictional force, $-m \gamma \mathbf{v}_{i}(t)$, and the random force, $\mathbf{R}_{i}(t)$. The former describes friction, while the latter describes random collisions with surrounding solvent molecules.

The two additional forces represent the interactions with the heat bath and are coupled through the friction coefficient:

$$
\begin{gathered}
\langle\mathbf{R}(t)\rangle=0 \\
\left\langle\mathbf{R}(t) \cdot \mathbf{R}\left(t^{\prime}\right)\right\rangle=2 k T m \gamma \delta\left(t-t^{\prime}\right)
\end{gathered}
$$

This is also known as the fluctuation-dissipation theorem.

The Langevin equation is a stochastic differential equation that is solved numerically and, therefore, approximately. Several algorithms exist in the literature for its integration [34-37].

Here, we employ the simple and effective algorithm of Grønbech-Jensen and Farago (GJF). The original version [24] had a Verlet-type formalism. Recent modifications by Farago (GJF-F) [25] and Grønbech Jensen and 
Table 1: Reduced quantities

\begin{tabular}{llll}
\hline Quantity & Symbol & Unit quantity & Reduced quantity \\
\hline Time & $t$ & $t_{0}=d \sqrt{\frac{m}{k T}}$ & $t^{*}=\frac{t}{d} \sqrt{\frac{k T}{m}}$ \\
Distance & $r$ & $r_{0}=d$ & $r^{*}=\frac{r}{d}$ \\
Density & $\rho$ & $\rho_{0}=\frac{1}{d^{3}}$ & $\rho^{*}=\rho d^{3}$ \\
Velocity & $v$ & $v_{0}=\frac{d}{t_{0}}=\sqrt{\frac{k T}{m}}$ & $v^{*}=v \sqrt{\frac{m}{k T}}$ \\
Energy & $u$ & $u_{0}=k T$ & $u^{*}=\frac{u}{k T}$ \\
Force & $F$ & $F_{0}=\frac{k T}{d}$ & $F^{*}=\frac{F d}{k T}$ \\
Dipole moment & $p$ & $p_{0}=\sqrt{4 \pi \epsilon_{0} k T d^{3}}$ & $p^{*}=\frac{p}{\sqrt{4 \pi \epsilon_{0} k T d^{3}}}$ \\
Friction coefficient & $\gamma$ & $\gamma_{0}=\frac{1}{t_{0}}=\frac{1}{d} \sqrt{\frac{k T}{m}}$ & $\gamma^{*}=\gamma t_{0}=\gamma d \sqrt{\frac{m}{k T}}$. \\
\hline
\end{tabular}

Grønbech-Jensen (GJF-2GJ) [26] have a leap-frog formalism using velocities in the half time steps. These modifications have the advantage that they accurately sample both kinetic and configurational properties even for large time steps within the stability limit. The authors demonstrated the efficiency of their algorithms for systems under linear and harmonic potentials. We use the GJF-2GJ version in this work that reads as

$$
\begin{gathered}
v^{n+\frac{1}{2}}=a v^{n-\frac{1}{2}}+\frac{\sqrt{b} \Delta t}{m} f^{n}+\frac{\sqrt{b}}{2 m}\left(R^{n}-R^{n+1}\right) \\
r^{n+1}=r^{n}+\sqrt{b} v^{n+\frac{1}{2}} \Delta t
\end{gathered}
$$

where $r^{n}=r\left(t^{n}\right)$ is any position coordinate of any particle, $v^{n}=v\left(t_{n}\right)$ is any velocity coordinate of any particle,

$$
\begin{aligned}
& a=\frac{1-\gamma \Delta t / 2}{1+\gamma \Delta t / 2}, \\
& b=\frac{1}{1+\gamma \Delta t / 2},
\end{aligned}
$$

$\Delta t$ is the time step, $t_{n+\frac{1}{2}}=t_{n}+\frac{\Delta t}{2}$, and $t_{n-\frac{1}{2}}=t_{n}-\frac{\Delta t}{2}$. The discrete time noise

$$
R^{n+1}=\int_{t_{n}}^{t_{n+1}} R\left(t^{\prime}\right) d t^{\prime}
$$

is a random Gaussian number with properties

$$
\left\langle R^{n}\right\rangle=0
$$

and

$$
\left\langle R^{m} R^{n}\right\rangle=2 k T \gamma m \Delta t \delta_{m n}
$$

with $\delta_{m n}$ being the Kronecker-delta.

\section{Scaling and reduced units}

Competing effects exist in an ER system. The DD interactions have an ordering effect. The head-to-tail position, in which the dipoles are aligned along $\mathbf{n}_{i j}(\theta=0)$ at contact $\left(r_{i j}=d\right)$, has a minimum energy with the value

$$
u_{0}=-\frac{1}{4 \pi \epsilon_{0}} \frac{2 p^{2}}{d^{3}} .
$$

The magnitude of the force in this position is

$$
f_{0}=\frac{3 p^{2}}{4 \pi \epsilon_{0} d^{4}} .
$$

The Brownian motion has a disordering effect that expresses the coupling to a thermostat of temperature $T$ and friction with the surrounding solvent with viscosity $\eta$. It is usual to characterize the disordering effect of the thermal motion energetically by $k T$. It is also usual to use reduced units in calculations. In reduced units our quantities are expressed as dimensionless numbers obtained by dividing a quantity in a physical unit by a unit quantity in the same unit, $t^{*}=t / t_{0}$, for example. Reduced quantities are useful not only because their values are close to 1 , so it is easier to work with them, but also because they express relations between quantities in the numerator and the denominator, a kind of scaling [5].

There are different ways of defining reduced units. We use the convention of building the unit quantities from the mass, $m$, the particle diameter, $d$, and $k T$. Thus, the reduced units collected in Table 1 can be defined.

When we perform simulations in reduced units, these quantities can be chosen freely to see how the system behaves at the different combinations of the reduced parameters. How the reduced parameters are related to real-life physical parameters can be computed independently (see Section 5). 
Table 2: Experimental parameters [38,39].

\begin{tabular}{ll}
\hline$\epsilon_{\text {in }}$ & 4 \\
$\epsilon_{\text {out }}$ & 2.7 \\
$\eta(\mathrm{Pa} \mathrm{s})$ & 0.5 \\
$E_{0}(\mathrm{~V} / \mathrm{m})$ & $10^{6}$ \\
$T(K)$ & 300 \\
$\rho_{\text {out }}\left(\mathrm{kg} / \mathrm{m}^{3}\right)$ & 2650 \\
\hline
\end{tabular}

The reduced quantities collected in Table 1 are determined by the real physical parameters of the system: the temperature, $T$, the mass density of the material of the ER particle, $\rho_{\text {in }}$, the diameter of the ER particle, $d$, the dielectric constant of the ER particle, $\epsilon_{\mathrm{in}}$, the dielectric constant of the solvent, $\epsilon_{\text {out }}$, the viscosity of the solvent, $\eta$, and the strength of the applied electric field, $E_{0}$. For a specific ER fluid, these variables are tabulated in Table 2. This specific example is used because one of the coauthors (I.SZ.) published experimental results for this system [38,39]. A wide variety of ER fluids exists, however.

The mass of a particle is computed as $m=\rho_{\text {in }} \pi d^{3} / 6$, so it scales with $d^{3}$. The dipole moment of a particle is given by Eq. 2 that shows that $p$ scales with $d^{3}$.

An important parameter is the ratio of the dipolar energy and the thermal energy that is expressed by the square of the reduced dipole moment:

$$
\left(p^{*}\right)^{2}=\frac{\pi \epsilon_{0} E_{0}^{2}}{4 k T}\left(\frac{\epsilon_{\text {in }}-\epsilon_{\text {out }}}{\epsilon_{\text {in }}+2 \epsilon_{\text {out }}}\right)^{2} d^{3}=K d^{3}
$$

that scales with $d^{3}$. If $p^{*}$ is large, the dipolar interactions are strong enough to induce chain formation. If $p^{*}$ is too large, the chains freeze, and the ER particles solidify (note that the fluid itself does not solidify). If $p^{*}$ is small, thermal motion prevents chain formation and/or breaks the chains.

The friction coefficient can be computed from Stokes' law as

$$
\gamma=\frac{3 \pi \eta d}{m}=\frac{18 \eta}{\rho_{\text {in }}} d^{-2}
$$

so it scales with $d^{-2}$. The value of $\gamma^{*}$ describes the strength of the coupling with the solvent and it scales with $d^{1 / 2}$. If $\gamma^{*}$ is large, friction and the disordering effect of the random force are strong. The diffusivity of the particles in the fluid, therefore, will be smaller. The diffusion constant in the high coupling limit can be expressed by Einstein's relation:

$$
D=\frac{k T}{m \gamma}
$$

or, in reduced units, $D^{*}=1 / \gamma^{*}$. If $\gamma^{*} \rightarrow 0$, the frictional and the random forces vanish, and the Langevin equation goes into the Newton equation. The particles move in vacuum without a thermostat; this practically corresponds to an MD simulation in the microcanonical ensemble. If $\gamma^{*}$ is small, we talk about an MD simulation with a Langevin thermostat.
In the case of the ER fluids, we are in the regime of large $\gamma^{*}$. As we will see, $\gamma^{*}$ is in the order of $10^{4}-10^{6}$. In this case, our concern is how to make the simulation efficient in order to collect enough information about the dynamics of the system in a reasonable amount of computer time.

The parameter with which we can tune the speed of sampling is the time step, $\Delta t^{*}$. This parameter is also subject of optimization. If $\Delta t^{*}$ is too small, the simulation will evolve slowly at the price of expensive computation time. If $\Delta t^{*}$ is too large, the spheres might overlap and the repulsive core force (Eq. 12) becomes so large that the particles shoot apart resulting in unphysical movements. This leads to instabilities in solving the Langevin equation.

Various solutions have been proposed in the literature to cope with this problem. If the Langevin integration algorithm allows changing the time step during the simulation, it is a reasonable suggestion to reduce the time step if we observe problems (generally, big jumps) in the movements of particles $[6,13]$. Displacements, velocities, or forces can be monitored for unusual events.

Berti et al. [40] used a uniform time step, while their solution for the jump-problem was that they went back the necessary number of time steps and started again with a different random number seed for the random force. If such a problem is rare, this can be a good solution, because the computational cost of going back a couple of times is balanced by the large time step used in the simulation. They used their simulations for ion channels whose selectivity filter is a high-density region, so overlaps can occur. Chain formation in the ER fluid also brings particles close to each other, so we need to be careful with large time steps.

We can estimate in advance the danger of overlap and judge the optimization between slow simulations (small $\Delta t$ ) and jumping particles (large $\Delta t$ ). We can introduce the average distance that a particle moves in a time step with the average thermal velocity, $\bar{v}=\sqrt{3 k T / m}$. Let us introduce

$$
\overline{\Delta s^{*}}=\frac{\bar{v} \Delta t}{d}=\sqrt{3} \Delta t^{*},
$$

that characterizes the average distance with respect to the particle size. This is proportional to $\Delta t^{*}$. This reduced distance, and, consequently, the reduced time step should be smaller than 1 . This imposes a strict limit to the time step.

The product $\gamma \Delta t=\gamma^{*} \Delta t^{*}$ characterizes how close we are to the overdamped limit. Basically, at a fixed $\gamma^{*}$, we can increase $\Delta t^{*}$ up to the threshold limit to save computer time at the price of losing information about dynamics due to coarser time resolution.

The last parameter that we can choose relatively freely is the energy parameter of the LJ potential, $\varepsilon^{\mathrm{LJ}}$, see Eqs. 10-12. Changing this parameter practically changes the effective diameter of the particles. Fig. 3 shows the curves of the core potential (Eq. 10) for varying values of $\varepsilon^{\mathrm{LJ}}$. Smaller values of $\varepsilon^{\mathrm{LJ}}$ allows for the particles to 


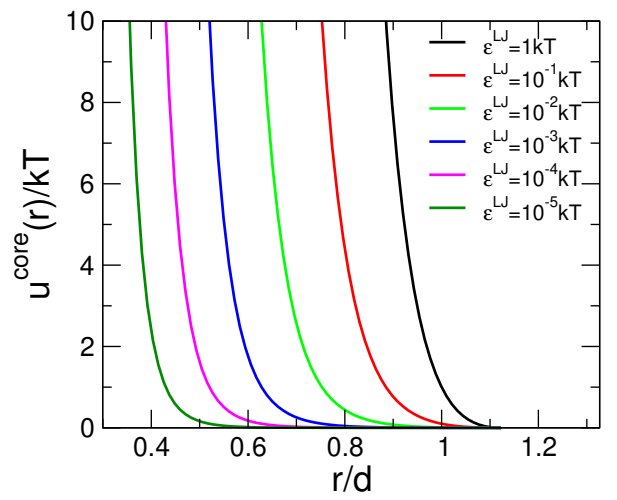

Figure 3: The core potential, $u^{\mathrm{WCA}}(r)$, for varying energy parameters, $\varepsilon^{\mathrm{LJ}}$.

approach each other closer: the $r / d$ values at which the core potential reaches large values in $k T$ are smaller for smaller $\varepsilon^{\text {LJ }}$ values. The effective diameter, $d_{\text {eff }}$, therefore decreases with decreasing $\varepsilon^{\mathrm{LJ}}$.

This results in larger dipole-dipole interactions at contact positions that, in turn, increases the weight of the dipolar interactions with respect to the thermal noise. Using smaller $\varepsilon^{\mathrm{LJ}}$, and, consequently, smaller $d_{\text {eff }}$, however, makes our parameter $d$ with which we reduced every variable meaningless. We would like the diameter used in the reduced quantities to be the real diameter of the spheres. For this reason, we do not change $\varepsilon^{\mathrm{LJ}}$ and fix it at the value of $k T$.

\section{Relating reduced units to real ER fluids}

To connect to a real system, we consider the ER fluid studied by Horváth and Szalai [38, 39] experimentally. The experimental parameters are collected in Table 2 . Note that the diameters used in these studies were quite small in order to prevent sedimentation. Diameters used in other ER fluids are larger reaching $1 \mu \mathrm{m}$.

We change two parameters in this analysis, the particle diameter, $d$, and the reduced time step, $\Delta t^{*}$. According to Eq. 2, the dipole moment can be written as $p=K d^{3}$, where $K=1.922 \times 10^{-6} \mathrm{Cm}^{-2}$ for the parameters in Table 2 . Table 3 contains various quantities computed for different values of $d$.

It is seen that $p^{*}$ falls into the regime simulated in this study around $d=1 \mu \mathrm{m}$. For diameters below $100 \mathrm{~nm}$, at least, at the present value of $K$, the reduced dipole moment is too weak to counterbalance the thermal motion and to produce considerable chain formation.

The reduced friction coefficient also depends on $d$; it increases with $d^{1 / 2}$. It is in the regime of $\gamma^{*} \approx 10^{5}-10^{6}$. This looks simulatable, though it will require considerable computer time, because $\Delta t^{*}$ is limited. The parameter $\overline{\Delta s^{*}}$ is the same for every diameter; it practically equivalent to $\Delta t^{*}$. To look at the effect of $\Delta t^{*}$, we show the same data for varying $\Delta t^{*}$ at a fixed $d(100 \mathrm{~nm})$ in Table 4.
Table 3: Change of various variables as the diameter of spheres is changed from 10 to $10,000 \mathrm{~nm}$ for time step $\Delta t^{*}=0.001$.

\begin{tabular}{l|rrrr}
\hline$d(\mathrm{~nm})$ & $\mathbf{4}$ & \multicolumn{4}{c}{$\mathbf{1 0 0}$} & $\mathbf{1 , 0 0 0}$ & $\mathbf{1 0 , 0 0 0}$ \\
$\Delta t^{*}$ & \multicolumn{4}{|c}{0.001} \\
\hline$m(\mathrm{~kg})$ & $1.387 \mathrm{E}-21$ & $1.387 \mathrm{E}-18$ & $1.387 \mathrm{E}-15$ & $1.387 \mathrm{E}-12$ \\
$t_{0}(\mathrm{~s})$ & $5.788 \mathrm{E}-09$ & $1.830 \mathrm{E}-06$ & $5.788 \mathrm{E}-04$ & $1.830 \mathrm{E}-01$ \\
$\bar{v}(\mathrm{~m} / \mathrm{s})$ & $2.993 \mathrm{E}+00$ & $9.463 \mathrm{E}-02$ & $2.993 \mathrm{E}-03$ & $9.463 \mathrm{E}-05$ \\
$p(\mathrm{Cm})$ & $1.922 \mathrm{E}-30$ & $1.922 \mathrm{E}-27$ & $1.922 \mathrm{E}-24$ & $1.922 \mathrm{E}-21$ \\
$p^{*}$ & $\mathbf{0 . 0 0 2 8 3}$ & $\mathbf{0 . 0 8 9 6}$ & $\mathbf{2 . 8 3 3}$ & $\mathbf{8 9 . 6 0}$ \\
$\Delta t(\mathrm{~s})$ & $5.788 \mathrm{E}-12$ & $1.830 \mathrm{E}-09$ & $5.788 \mathrm{E}-07$ & $1.830 \mathrm{E}-04$ \\
$\Delta s^{*}$ & 0.00173 & 0.00173 & 0.00173 & 0.00173 \\
$\gamma(1 / \mathrm{s})$ & $3.396 \mathrm{E}+13$ & $3.396 \mathrm{E}+11$ & $3.396 \mathrm{E}+09$ & $3.396 \mathrm{E}+07$ \\
$\gamma^{*}$ & $1.966 \mathrm{E}+05$ & $6.216 \mathrm{E}+05$ & $1.966 \mathrm{E}+06$ & $6.216 \mathrm{E}+06$ \\
$\gamma \Delta t$ & $1.966 \mathrm{E}+02$ & $6.216 \mathrm{E}+02$ & $1.966 \mathrm{E}+03$ & $6.216 \mathrm{E}+04$ \\
\hline
\end{tabular}

Table 4: Change of various variables as the reduced time step $\Delta t^{*}$ is changed from 0.0001 to 0.1 for diameter $d=$ $100 \mathrm{~nm}$.

\begin{tabular}{l|rrrr}
\hline$d(\mathrm{~nm})$ & \multicolumn{4}{|c}{100} \\
$\Delta t^{*}$ & $\mathbf{0 . 0 0 0 1}$ & $\mathbf{0 . 0 0 1}$ & $\mathbf{0 . 0 1}$ & $\mathbf{0 . 1}$ \\
\hline$m(\mathrm{~kg})$ & $1.387 \mathrm{E}-18$ & $1.387 \mathrm{E}-18$ & $1.387 \mathrm{E}-18$ & $1.387 \mathrm{E}-18$ \\
$t_{0}(\mathrm{~s})$ & $1.830 \mathrm{E}-06$ & $1.830 \mathrm{E}-06$ & $1.830 \mathrm{E}-06$ & $1.830 \mathrm{E}-06$ \\
$\bar{v}(\mathrm{~m} / \mathrm{s})$ & $9.463 \mathrm{E}-02$ & $9.463 \mathrm{E}-02$ & $9.463 \mathrm{E}-02$ & $9.463 \mathrm{E}-02$ \\
$p(\mathrm{Cm})$ & $1.922 \mathrm{E}-27$ & $1.922 \mathrm{E}-27$ & $1.922 \mathrm{E}-27$ & $1.922 \mathrm{E}-27$ \\
$p^{*}$ & 0.0896 & 0.0896 & 0.0896 & 0.0896 \\
$\Delta t$ & $1.830 \mathrm{E}-10$ & $1.830 \mathrm{E}-09$ & $1.830 \mathrm{E}-08$ & $1.830 \mathrm{E}-07$ \\
$\Delta s^{*}$ & $\mathbf{0 . 0 0 0 1 7 3}$ & $\mathbf{0 . 0 0 1 7 3}$ & $\mathbf{0 . 0 1 7 3}$ & $\mathbf{0 . 1 7 3}$ \\
$\gamma(1 / \mathrm{s})$ & $3.396 \mathrm{E}+11$ & $3.396 \mathrm{E}+11$ & $3.396 \mathrm{E}+11$ & $3.396 \mathrm{E}+11$ \\
$\gamma^{*}$ & $6.216 \mathrm{E}+05$ & $6.216 \mathrm{E}+05$ & $6.216 \mathrm{E}+05$ & $6.216 \mathrm{E}+05$ \\
$\gamma \Delta t$ & $6.216 \mathrm{E}+01$ & $6.216 \mathrm{E}+02$ & $6.216 \mathrm{E}+03$ & $6.216 \mathrm{E}+04$ \\
\hline \multicolumn{5}{l}{}
\end{tabular}

\section{Results and Discussion}

In this study, we use a relatively small number of particles $(N=128)$ in order to save on computer time and be able to explore a wide range of parameters in reduced units. We also fix the packing fraction expressed in term of the reduced density at $\rho^{*}=0.05$. At these values the width of the simulation cell is $L=13.68 \mathrm{~d}$.

The computer code has been written (in Fortran) in a way that we perform $M_{0}$ time steps in the absence of applied electric field $\left(\mathbf{E}_{0}=0\right)$, and $M_{\mathrm{E}}$ time steps in the presence of it. That way, we can study the dynamics of chain formation after the electric field is switched on. To improve statistics, we can perform several of this $M_{\mathrm{c}}=$ $M_{0}+M_{\mathrm{E}}$ cycles and average over the cycles.

When we start a cycle over, we can choose between two options. We can either continue the simulation from the previous phase state point (configurations and velocities) only without dipoles, or we can restart from a freshly generated initial configuration. In this work, we choose the second option. This choice ensures that we start the simulation with nonzero $E_{0}$ in a completely disordered state without chains. The first option makes it possible to study the dynamics of the deconstruction of the chains. 

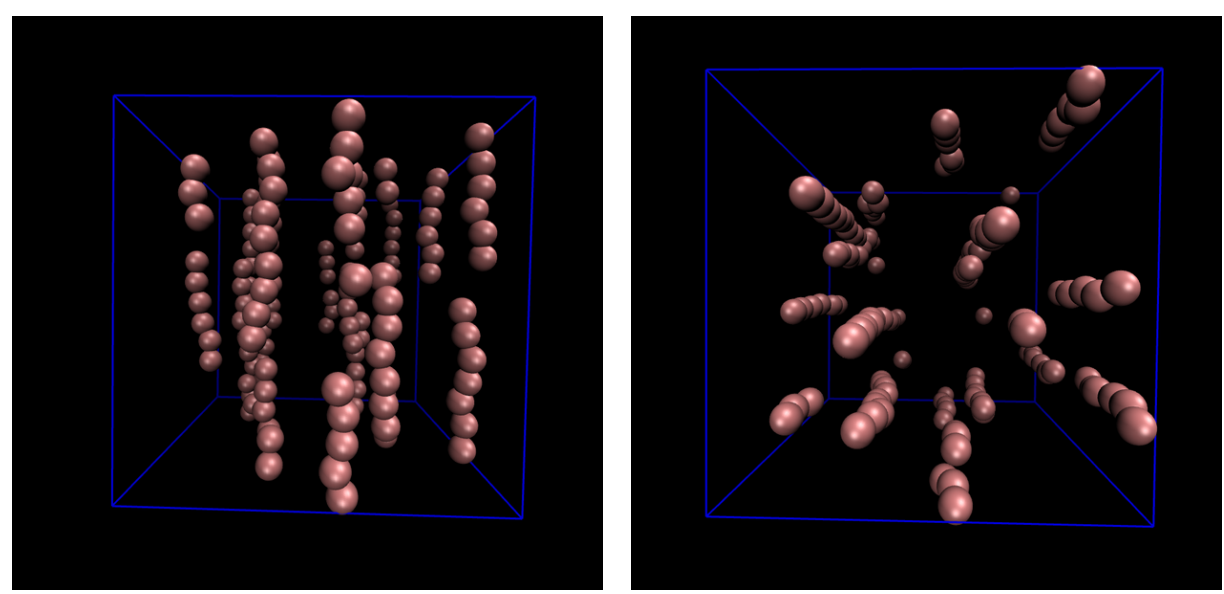

Figure 4: Typical snapshot of a simulation from the front (perpendicular to the $z$ axis, left panel) and top (parallel to the $z$ axis, right panel) for a state when chains are formed.

\subsection{Quantities studied}

As the chains are being formed, certain physical quantities change, so they directly or indirectly characterize chain formation quantitatively. In chains, particles are aligned into head-to-tail position along the $z$-axis as shown in Fig. 4. There are longer and shorter chains and the distribution of chains of various lengths changes continuously as the simulation evolves.

Since the head-to-tail position is the lowest energy configuration of the ER spheres (see Fig. 2 and Eqs. 24 and 25), the average one-particle dipole-dipole energy, $\left\langle u^{\mathrm{DD}}\right\rangle_{\mathrm{b}} / k T$, is a good indicator of chain formation. As it turns out, it is the best converging indicator.

By average, we mean average over a block in the simulation, denoted by $\langle\ldots\rangle_{\mathrm{b}}$. The length of a block $\left(M_{\mathrm{b}}\right.$ is the number of time steps in a block), again, is a subject of optimization. If a block is too short, the physical quantities averaged over a block will have bad statistics. If a block is too long, we loose information about the dynamics of the system.
Diffusion constant When the particles are "frozen" into chains, their mobility decreases. Chains are frozen only at very large dipole moments, when even columnar structures are formed. In a moderate range of $\left(p^{*}\right)^{2}$, chains move around, break apart, and rejoin, see the video clip at https://youtu.be/OwXsuz6pow4. A snapshot of this video clip is shown in Fig. 5.

The isotropic diffusion constant is computed as the slope of the mean square displacement (MSD) as a function of time:

$$
D_{\mathrm{b}}=\frac{\left\langle\mathbf{r}^{2}(t)\right\rangle_{\mathrm{b}}}{2 t_{\mathrm{b}}}
$$

where $\langle\ldots\rangle_{\mathrm{b}}$ denotes an average over time steps in a block and particles and $t_{\mathrm{b}}$ is the length of the block in time. The exact equilibrium diffusion constant is obtained in the limit of $t_{\mathrm{b}} \rightarrow \infty$.

Here, we must be satisfied with an approximate value of $D_{\mathrm{b}}$ obtained over a block of limited length. Fig. 6 shows the MSD as a function of $t^{*}$ for six equidistantly chosen blocks. In this particular case, $\gamma^{*}=5000$, so the slope is $D^{*}=\mathrm{MSD} / t_{\mathrm{b}}^{*} \approx 0.0002$ for the WCA fluid as

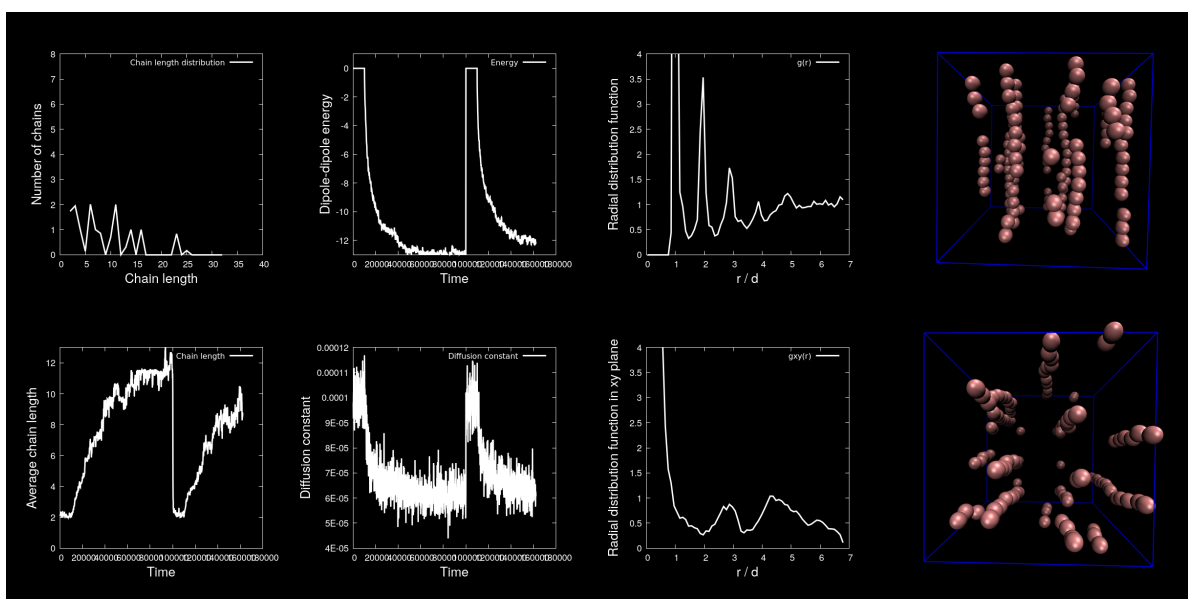

Figure 5: A snapshot of the video clip at https://youtu.be/OwXsuz 6p0w4. 


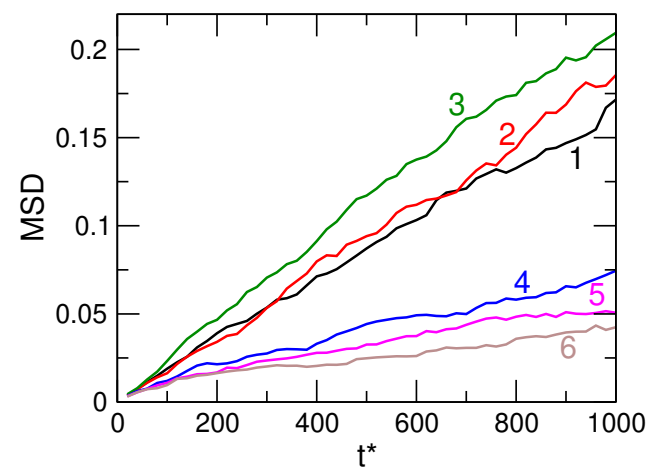

Figure 6: The mean square displacement for six selected blocks. The blocks are selected in equidistant time periods in way that the first three belong to the $E=0$ phase, while the second three belongs to the ER phase. Parameters: $\left(p^{*}\right)^{2}=6, \gamma^{*}=5,000, \Delta t^{*}=0.02, M_{\mathrm{b}}=50,000$.

also expressed by the Einstein relation $\left(D^{*}=1 / \gamma^{*}\right.$, Eq. 28). Here, the time-length of the block is $t_{\mathrm{b}}^{*}=\Delta t^{*} M_{\mathrm{b}}=$ 1,000 , because $\Delta t^{*}=0.02$ and $M_{\mathrm{b}}=50,000$. The first three lines are in the $E=0$ regime, while the second three lines are in the ER regime. The slope apparently is smaller in the ER case than in the WCA case, but the scattering is large.

The sampling can be improved by averaging over cycles, but this does not help on the problem of the diffusion constant being approximate obtained for a too short block.

Chain length distributions The chain formation can be directly followed by identifying chains in every configuration. If that is done, we can obtain the number of chains, $n_{s}$, having length $s$. The average chain length can be computed as

$$
l=\frac{\sum_{s} s n_{s}}{\sum_{s} n_{s}}
$$

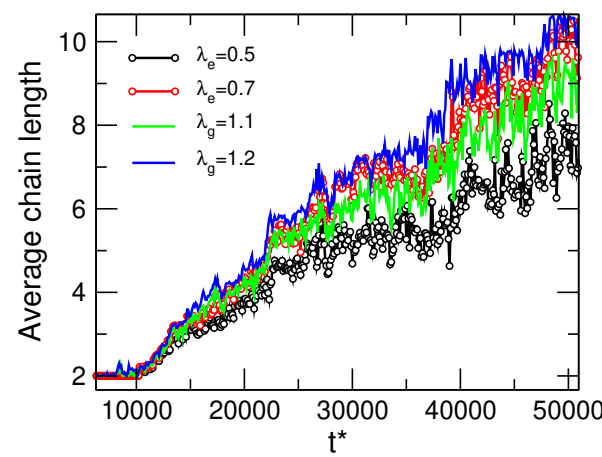

Figure 7: The trend of the change in the average chain length with various definitions of a chain: energetic with $\lambda_{\mathrm{e}}=0.5$ and 0.7 , geometrical with $\lambda_{\mathrm{g}}=1.1$ and 1.2. Parameters: $\left(p^{*}\right)^{2}=6, \gamma^{*}=10,000, \Delta t^{*}=0.01$, $M_{\mathrm{b}}=10,000$.

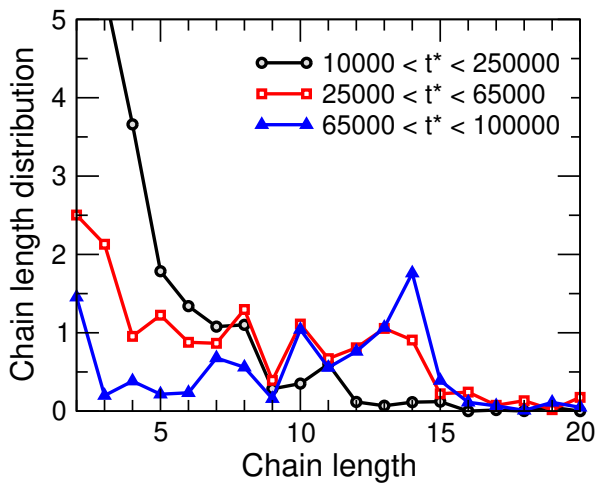

Figure 8: Chain length distribution averaged over three time intervals at the beginning $\left(10,000<t^{*}<25,000\right)$, in the middle $\left(25,000<t^{*}<65,000\right)$, and at the end $\left(65,000<t^{*}<100,000\right)$ of chain formation. Parameters are the same as at Fig. 7.

This quantity than can be averaged over time steps in a block, so chain formation can be followed by plotting the average chain length, $\langle l\rangle_{\mathrm{b}}$, as a function of time in steps of $t_{\mathrm{b}}^{*}$.

A chain, however, can be defined in various ways. One simple definition is geometrical. If two particles are closer to each other than a predefined distance:

$$
r_{i j}<\lambda_{\mathrm{g}} d,
$$

they are said to be part of the same chain. Another definition is energetic. If the dipole-dipole interaction energy is smaller than a predefined threshold:

$$
u_{i j}^{\mathrm{DD}}\left(r_{i j}, \theta\right)<\lambda_{\mathrm{e}} u_{0},
$$

then they are said to be part of the same chain, where $u_{0}$ is the DD interaction energy in the head-to-tail position (Eq. 24).

Fig. 7 shows the increase of the average chain length as a function of time as obtained from different chain definitions and thresholds $\lambda_{\mathrm{e}}$ and $\lambda_{\mathrm{g}}$. In general, the trends as shown by the various definitions are the same. The dynamic process of chains breaking up and reforming have the same effect in the cases of the various definitions. This process can be characterized by time constants obtained from fitting exponential functions. These time constants are insensitive to the choice of the chain definition. Here, we will use the geometrical definition with the parameter $\lambda_{\mathrm{g}}=1.2$. The geometrical definition is advantageous, because it can also be used in the absence of an electric field.

The average chain length is an informative, but averaged quantity. From the simulations, we have the more detailed $n_{s}$ vs. $s$ chain length distributions that give the average number of chains of different lengths as a function of $s$. This function varies with time, see the video clip at https://youtu.be/OwXsuz6pow4. To show the dynamics of this function, we average it for three distinct time intervals. The first one refers to the 


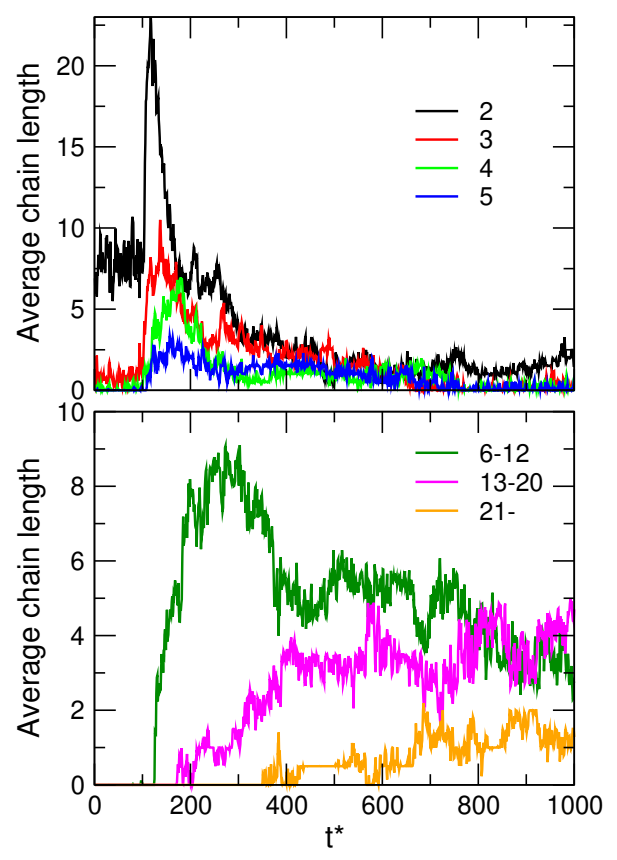

Figure 9: The variation of the number of chains of various lengths in time. Top: chains of lengths $2,3,4$, and 5 . Bottom: number of chains belonging to the ranges $6-12$, $13-20$, and above 21. Parameters are the same as at Fig. 7.

beginning of the time period in the presence of the field when the chains start forming. In the second, intermediate time interval $\left(25,000<t^{*}<65,000\right)$ longer chains are formed, while in the third time interval $(65,000<$ $\left.t^{*}<100,000\right)$, full chains crossing the simulation box are formed.

Fig. 8 shows these three time-averaged functions. At the beginning, there are many pairs and short chains (black curve). In the intermediate time interval, the number of short chains decreases and longer chains are formed. In the third time interval, a well-defined peak at $s=14$ appears that corresponds to the full chains crossing the simulation box of length $L=13.68 d$.

We can get a much better impression of the dynamics of chain formation, if we plot $n_{s}$ as a function of time. Because there are too many possible $n_{s}$ numbers to plot, again, we average over certain regions of chain lengths as seen in Fig. 9. In the top panel, the behavior of short chains from pairs to $s=5$ is shown. The behavior of these chains is qualitatively similar. First, as the electric field is switched on, their numbers increase abruptly, then, as longer chains absorb them, or they fuse into longer chains, their numbers gradually decreases. Practically, they behave like reactive intermediates in chemical reactions: their formation is a first necessary step towards the formation of the end products.

The number of chains whose lengths are between 6 and 12 (bottom panel) behaves similarly. The curve for the chains whose lengths are between 13 and 20, however, saturates around $n_{s}=4$. This means that there are

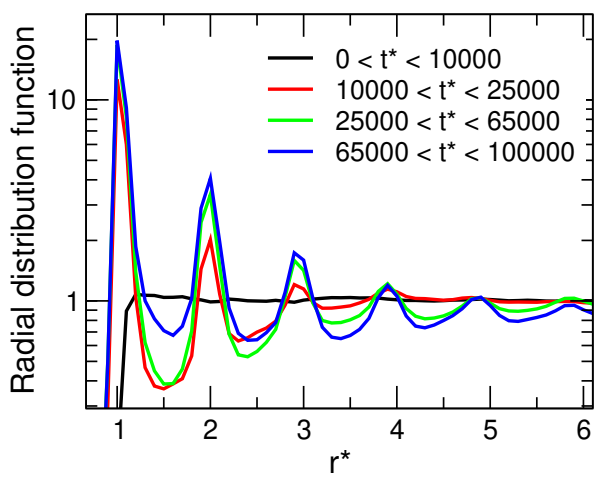

Figure 10: Radial distribution functions averaged over four time intervals in the absence of the electric field $(0<$ $\left.t^{*}<10,000\right)$, at the beginning $\left(10,000<t^{*}<25,000\right)$, in the middle $\left(25,000<t^{*}<65,000\right)$, and at the end $\left(65,000<t^{*}<100,000\right)$ of chain formation. Parameters are the same as at Fig. 7 .

generally about 4 full chains in the simulation box (this value, of course, depends on system size and packing fraction). They are often accompanied by shorter chains as seen in Fig. 4 and the video clip.

Chains longer than 20 also exist. It can also occur that two chains are stuck together. Whether it is a stable, long time-span configuration, depends on the strength of the dipole moment (the electric field, in reality). A particle is attracted to another particle in a chain, if they are aligned in a way that $\theta=\pi / 4$, see Fig. 2. This is a relatively weak attraction compared to the head-to-tail position. The chains displace due to thermal motion, so the chains move out of these mutual positions that favors aggregation of chains. If two chains move in a way that the particles get next to each other $(\theta=\pi / 2)$, a repulsive force replaces the weak attractive one. So, a strong dipole moment is needed to overcome the thermal motion if we want to see stable columnar aggregations of chains as seen many times in the literature.

Pair distribution functions As particles aggregate into chains, the structure of the fluid, generally expressed with pair distribution functions, changes. In an anisotropic dipolar fluid, we generally use the series expansion of the pair correlation function of axially symmetric molecules as

$$
g(i j)=\sum_{n m l} h^{m n l}\left(r_{i j}\right) u^{m n l}(i j) .
$$

This expansion separates distance and angular dependence in such a way that the projections $h^{m n l}\left(r_{i j}\right)$ depend only on the distance of particles and the projections $u^{m n l}(i j)$ are rotational invariants.

The projection $g\left(r_{i j}\right)=h^{000}\left(r_{i j}\right)$ is the usual radial distribution function (RDF):

$$
g\left(r_{i j}\right)=\int g(i j) d \Omega_{i} d \Omega_{j}, \quad \text { with } \quad u^{000}=1,
$$




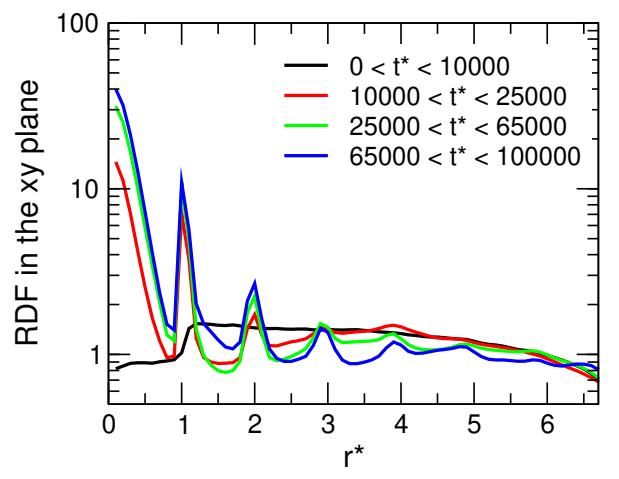

Figure 11: The $x y$-plane radial distribution functions averaged over four time intervals as in Fig. 10. Parameters are the same as at Fig. 7.

where $\Omega_{i}$ denotes molecular orientation. In a fluid phase, $h^{000}\left(r_{i j}\right) \rightarrow 1$ when $r_{i j} \rightarrow \infty$ both in isotropic and anisotropic phases. Other projections, called angular correlation functions, can also characterize chain formation, but we will discuss only the RDF in this study.

Similar to Fig. 8, we plot the RDF averaged over the time intervals discussed at the chain length distributions. In addition to those three time intervals, we also consider the time interval $0<t^{*}<10,000$ here, which is the time of the electric field being switched off. Fig. 10 shows that the $g(r)$ function behaves like a typical RDF for a dense real gas $\left(\rho^{*}=0.05\right)$ in the absence of $E_{0}$.

As the electric field is switched on, however, larger and larger peaks appear as time goes by and longer and longer chains are formed. The peaks appear at every integer multiples of $d$ values that correspond to particles in the chain. A more detailed behavior of $g(r)$ can be followed in the video clip: https: / / youtu.be/ OwXsuz 6p0w4.

When the chains are formed, they are relatively stable, but they diffuse around in the $x y$ plane. Therefore, we also define the RDF in the $x y$ plane to follow how the chains are distributed over the $x y$ plane. We will denote it with $g_{x y}(r)$ and is calculated the same way as the threedimensional RDF.

Fig. 11 shows these functions averaged over the time periods as in Fig. 10. A similar conclusion can be drawn as from that figure except that the first peak now appears at $r^{*}=0$, where now $r=\sqrt{\Delta x^{2}+\Delta y^{2}}$. This peak represents particles belonging to the same chain. Peaks represent probable distances between chains. The shape of the curve indicates that this ER system $\left(\left(p^{*}\right)^{2}=6\right)$ behaves like a two-dimensional fluid of chains.

At a given time (or, in a given block), these series of peaks are absent. Snapshots of $g_{x y}(r)$ show where the chains are in a given moment. This can be followed in the video clip: https://youtu.be/OwXsuz6p0W4. The $g_{x y}(r)$ function averaged over a longer time period characterizes the behavior of the chains as a twodimensional fluid.

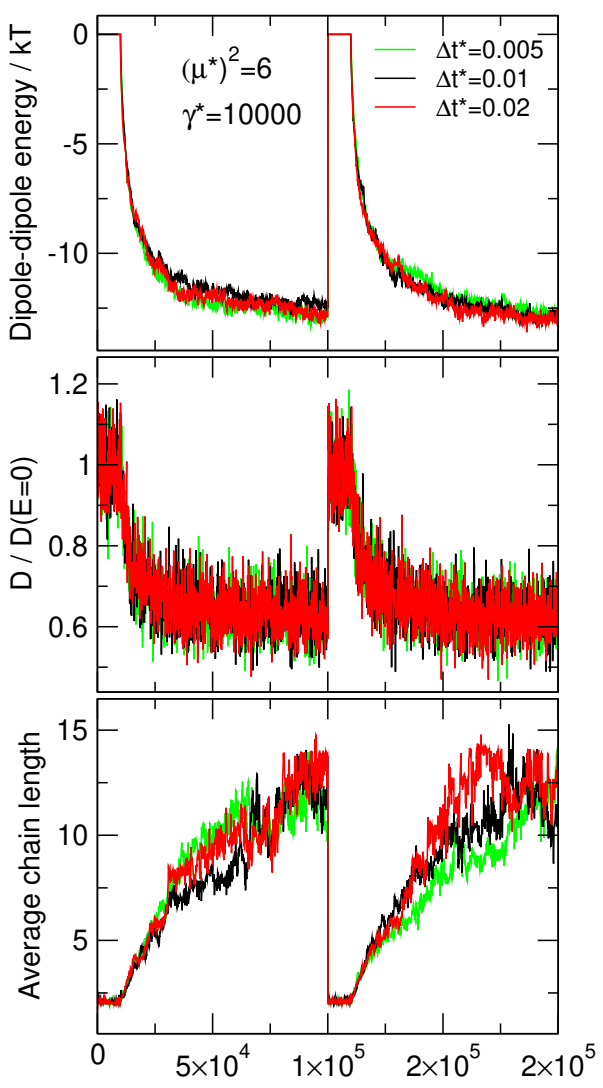

Figure 12: The one-particle dipole-dipole energy (top panel), the diffusion constant relative to its value in the absence of $E_{0}$ (middle panel), and the average chain length (geometrical definition with $\lambda_{\mathrm{g}}=1.2$, bottom panel) as functions of time using different time steps. Parameters: $\left(p^{*}\right)^{2}=6$ and $\gamma^{*}=10,000$. The $M_{\mathrm{b}}$ is changed in a way that $\Delta t^{*} \times M_{\mathrm{b}}$ is constant.

\subsection{The effect of time step}

First, let us consider the effect of the choice of the time step, $\Delta t^{*}$. Fig. 12 shows the variation of the one-particle dipole-dipole energy, the diffusion constant, and the average chain length (geometrical definition with $\lambda_{\mathrm{g}}=1.2$ ) for different values of $\Delta t^{*}$. The length of a block measured in $t^{*}$ is kept fixed. It is seen that the measured quantities behave the same way as a function of time, which indicates that the BD simulation algorithm is robust and provides results that are independent of the time step.

Also, we monitored the temperature computed from the kinetic energy, $\langle T\rangle=m\left\langle v^{2}\right\rangle / 3 k$, and found that the algorithm reproduces the prescribed temperature very precisely even for this highly anisotropic fluid. This supports the claim of the developers that this algorithm provides a very good Langevin thermostat [24-26].

If we change $\Delta t^{*}$, but we keep $M_{\mathrm{b}}$ at the same value, meaning that we change the time length of the block, the dipole-dipole energy and the average chain length are still insensitive to the choice of $\Delta t^{*}$ (data not shown). The diffusion coefficient, however, changes with the length of the blocks as already discussed above (at Fig. 6). This 


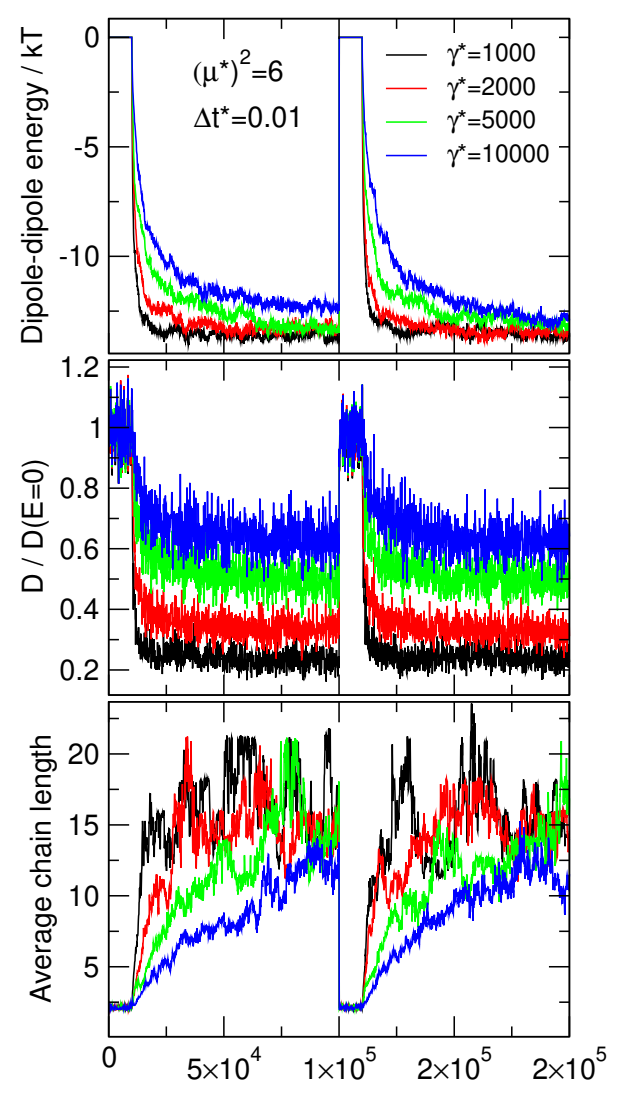

Figure 13: The one-particle dipole-dipole energy (top panel), the diffusion constant relative to its value in the absence of $E_{0}$ (middle panel), and the average chain length (geometrical definition with $\lambda_{\mathrm{g}}=1.2$, bottom panel) as functions of time using different friction coefficients. $\mathrm{Pa}$ rameters: $\left(p^{*}\right)^{2}=6$ and $\Delta t^{*}=0.01$.

means that we have a trade-off between satisfactory sampling over a block and good resolution in time.

We do not consider viscosity in this paper; we refer it to future studies. This trade-off will be present in the case of the viscosity (and the stress tensor) as well. It will be even more serious, because the viscosity is even a more poorly converging quantity than the diffusion constant.

\subsection{The effect of friction coefficient}

We fix the dipole moment at $\left(p^{*}\right)^{2}=6$ and the time step at $\Delta t^{*}=0.01$, and change the friction coefficient from $\gamma^{*}=1,000$ to 10,000 . As discussed in the next section, realistic ER fluids have friction coefficients even larger than 10,000 , but we refer studying that regime to future publications.

As $\gamma^{*}$ is increased, the curves tend to their equilibrium values as $E_{0}$ is switched on at a lower rate. Fitting exponential functions to these curves and identifying processes of different time lengths as parts of the complex process of chain formation will also be the subject of future studies.

The change in $\gamma^{*}$ does not influence the value where the energy and the average chain length converge to.

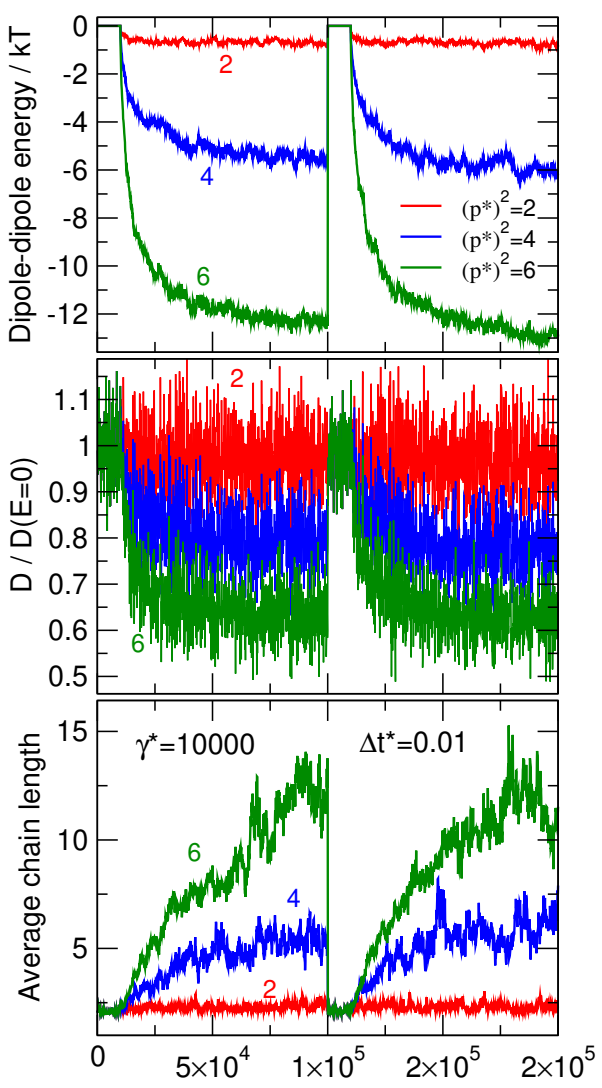

Figure 14: The one-particle dipole-dipole energy (top panel), the diffusion constant relative to its value in the absence of $E_{0}$ (middle panel), and the average chain length (geometrical definition with $\lambda_{\mathrm{g}}=1.2$, bottom panel) as functions of time using different dipole moments. Parameters: $\gamma^{*}=10,000$ and $\Delta t^{*}=0.01$. This figure shows two $M_{0}+M_{E}$ cycles.

They converge to the same value but with a different rate. Changing friction, however, changes the diffusion constant. Fig. 13 shows the diffusion constant relative to its value in the absence of the field computed as $D^{*}=1 / \gamma^{*}$. The diffusion constant decreases to a smaller value relative to $D^{*}(E=0)$ at smaller values of $\gamma^{*}$.

The average chain length shows that smaller $\gamma^{*}$ results in a more wildly fluctuating system than a larger $\gamma^{*}$. The particles diffuse faster and produce larger variations in configurations during a given time period.

\subsection{The effect of dipole moment}

Our simulations show (Fig. 14) that the quantity that determines the structure of the ER fluid is the reduced dipole moment, namely, the relation of the dipole-dipole energy to the thermal energy unit, $k T$. Fig. 14 shows that these quantities converge to their equilibrium values exhibiting a similar trend.

The dipole moments studied in this work belong to the regime where the ER fluid considered as a collection of chains is still a fluid, namely, it does not solidify. Several papers in the literature study solidification of the ER 
chains $[5,6,15,16,22]$.

\section{Summary}

In this work, we use a newly developed integrator algorithm to solve the Langevin equations and to perform BD simulations for ER fluids. Our focus was on the methodological development and identifying appropriate system parameters through which we can follow the dynamics of chain formation in the system.

The usefulness of computer simulations lies not only in the fact that we can follow the particles' trajectories, but also in the fact that we can gain a profound amount of information from these trajectories. In the BD simulations, for example, we can follow how the average number of chains of varying lengths changes in time. From that detailed information we can deduce time constants for characteristic processes during chain formation.

We intend to dig into those details in subsequent studies. Also, we want to examine the behavior of the chains under a stress.

\section{Acknowledgement}

This research was supported by the European Union, cofinanced by the European Social Fund. EFOP-3.6.2-162017-00002. We also acknowledge the support of the National Research, Development and Innovation Office NKFIH K124353.

\section{REFERENCES}

[1] Winslow, W.M.: Induced Fibration of Suspensions, J. Appl. Phys., 1949, 20(12), 1137-1140 DOI: $10.1063 \% 2 \mathrm{~F} 1.1698285$

[2] Duclos, T.G.; Carlson, J.D.; Chrzan, M.J.; Coulter, J.P.: Electrorheological Fluids - Materials and Applications, in Solid Mechanics and Its Applications (Springer Netherlands), 1992, 13, 213-241 DOI: 10.1007\%2F978-94-017-1903-2_5

[3] Havelka, K.O.; Filisko, F.E. (eds.): Progress in Electrorheology (Springer US), 1995. DOI: 10.1007\%2F978-1-4899-1036-3

[4] Klingenberg, D.J.; van Swol, F.; Zukoski, C.F.: Dynamic simulation of electrorheological suspensions, J. Chem. Phys., 1989, 91(12), 7888-7895 DOI: $10.1063 \% 2 \mathrm{~F} 1.457256$

[5] Heyes, D.M.; Melrose, J.R.: Brownian Dynamics Simulations of Electro-Rheological Fluids, II: Scaling Laws, Mol. Sim., 1990, 5(5), 293-306 DOI: 10.1080\%2F08927029008022415

[6] Whittle, M.: Computer simulation of an electrorheological fluid, J. Non-Newtonian Fluid Mechanics, 1990, 37(2-3), 233-263 DOI: 10.1016\%2F03770257\% 2890\%2990007-x

[7] Klingenberg, D.J.; Zukoski, C.F.: Studies on the steady-shear behavior of electrorheological suspensions, Langmuir, 1990, 6(1), 15-24 DOI: $10.1021 \% 2$ Fla00091a003
[8] Jaggi, N.K.: Structure and dynamics of a dense dipolar system in an electric field and their relevance to electrorheological fluids, J. Stat. Phys., 1991, 64(5-6), 1093-1102 DOI: 10.1007\%2Fbf01048816

[9] See, H.; Doi, M.: Aggregation Kinetics in ElectroRheological Fluids, J. Phys. Soc. Japan, 1991, 60(8), 2778-2782 DOI: 10.1143\%2Fjpsj.60.2778

[10] Bonnecaze, R.T.; Brady, J.F.: Dynamic simulation of an electrorheological fluid, J. Chem. Phys., 1992, 96(3), 2183-2202 DOI: 10.1063\%2F1.462070

[11] Toor, W.R.: Structure Formation in Electrorheological Fluids, J. Colloid Interf. Sci., 1993, 156(2), 335349 DOI: $10.1006 \% 2$ Fjcis.1993.1121

[12] Hass, K.C.: Computer simulations of nonequilibrium structure formation in electrorheological fluids, Phy. Rev. E, 1993, 47(5), 3362-3373 DOI: 10.1103\%2Fphysreve.47.3362

[13] Tao, R.; Jiang, Q.: Simulation of structure formation in an electrorheological fluid, Phys. Rev. Lett., 1994, 73(1), 205-208 DOI: 10.1103\%2Fphysrevlett.73.205

[14] Tao, R.; Jiang, Q.: Simulation of Solid Structure Formation in an Electrorheological Fluid, Int. J. Modern Phys. B, 1994, 08(20n21), 2721-2730 DOI: $10.1142 \% 2 F s 0217979294001081$

[15] Baxter-Drayton, Y.; Brady, J.F.: Brownian electrorheological fluids as a model for flocculated dispersions, J. Rheology, 1996, 40(6), 1027-1056 DOI: $10.1122 \% 2 \mathrm{~F} 1.550772$

[16] Gulley, G.L.; Tao, R.: Structures of an electrorheological fluid, Phys. Rev. E, 1997, 56(4), 4328-4336 DOI: $10.1103 \% 2$ Fphysreve. 56.4328

[17] Jian, L.; Jiapeng, S.: Simulation of a threedimensional electrorheological suspension, J. Appl. Phys., 1996, 79(9), 7312-7317 DOI: $10.1063 \% 2 \mathrm{~F} 1.361447$

[18] Wang, B.; Liu, Y.; Xiao, Z.: Dynamical modelling of the chain structure formation in electrorheological fluids, Int. J. Eng. Sci., 2001, 39(4), 453-475 DOI: $10.1016 \% 2 F s 0020-7225 \% 2800 \% 2900054-9$

[19] Enomoto, Y.; Oba, K.: Simulation of structures and their rheological properties in electrorheological fluids, Physica A, 2002, 309(1-2), 15-25 DOI: 10.1016\%2Fs0378-4371\%2802\%2900599-x

[20] Climent, E.; Maxey, M.R.; Karniadakis, G.E.: Dynamics of Self-Assembled Chaining in Magnetorheological Fluids, Langmuir, 2004, 20(2), 507513 DOI: $10.1021 \% 2$ Fla $035540 z$

[21] Cao, J.G.; Huang, J.P.; Zhou, L.W.: Structure of Electrorheological Fluids under an Electric Field and a Shear Flow: Experiment and Computer Simulation, J. Phys. Chem. B, 2006, 110(24), 11635 11639 DOI: $10.1021 \% 2 \mathrm{Fjp} 0611774$

[22] Domínguez-García, P.; Melle, S.; Pastor, J.M.; Rubio, M.A.: Scaling in the aggregation dynamics of a magnetorheological fluid, Phys. Rev. E, 2007, 76(5), 051403 DOI: $10.1103 \% 2 F p h y s r e v e .76 .051403$

[23] Heyes, D.M.: Rheology of molecular liquids and concentrated suspensions by microscopic dynami- 
cal simulations, J. Non-Newton. Fluid, 1988, 27(1), 47-85 DOI: $10.1016 \% 2 F 0377-0257 \% 2888 \% 2980004-1$

[24] Grønbech-Jensen, N.; Farago, O.: A simple and effective Verlet-type algorithm for simulating Langevin dynamics, Mol. Phys., 2013, 111(8), 983991 DOI: $10.1080 \% 2$ F00268976.2012.760055

[25] Farago, O.: Langevin thermostat for robust configurational and kinetic sampling, Physica A, 2019, 534, 122210 DOI: $10.1016 \% 2 F j$.physa.2019.122210

[26] Jensen, L.F.G.; Grønbech-Jensen, N.: Accurate configurational and kinetic statistics in discrete-time Langevin systems, Mol. Phys., 2019, 117(18), 2511-2526 DOI: 10.1080\%2F00268976.2019.1570369

[27] Sherman, S.G.; Paley, D.A.; Wereley, N.M.: Massively Parallel Simulations of Chain Formation and Restructuring Dynamics in a Magnetorheological Fluid, in ASME 2011 Conference on Smart Materials, Adaptive Structures and Intelligent Systems, Volume 1 (ASMEDC), DOI: 10.1115\%2Fsmasis20115188

[28] Sherman, S.G.; Paley, D.A.; Wereley, N.M.: Parallel Simulation of Transient Magnetorheological Direct Shear Flows Using Millions of Particles, IEEE Transactions on Magnetics, 2012, 48(11), 35173520 DOI: 10.1109\%2Ftmag.2012.2201214

[29] Fernández-Toledano, J.C.; Ruiz-López, J.A.; Hidalgo-Álvarez, R.; de Vicente, J.: Simulations of polydisperse magnetorheological fluids: A structural and kinetic investigation, J. Rheology, 2015, 59(2), 475-498 DOI: 10.1122\%2F1.4906544

[30] Boda, D.; Valiskó, M.; Szalai, I.: The origin of the interparticle potential of electrorheological fluids, Cond. Matt. Phys., 2013, 16(4), 43002 DOI: 10.5488/cmp. 16.43002

[31] Jackson, J.D.: Classical Electrodynamics (Wiley, New York), 3rd edn., 1999. ISBN: 978-0471309321

[32] Boda, D.; Gillespie, D.; Nonner, W.; Henderson, D.; Eisenberg, B.: Computing induced charges in inhomogeneous dielectric media: Application in a
Monte Carlo simulation of complex ionic systems, Phys. Rev. E, 2004, 69(4), 046702 DOI: 10.1103/physreve.69.046702

[33] Lemons, D.S.; Gythiel, A.: Paul Langevin's 1908 paper "On the Theory of Brownian Motion" ["Sur la théorie du mouvement brownien," C. R. Acad. Sci. (Paris) 146, 530-533 (1908)], Am. J. Phys., 1997, 65(11), 1079-1081 DOI: 10.1119\%2F1.18725

[34] Schneider, T.; Stoll, E.: Molecular-dynamics study of a three-dimensional one-component model for distortive phase transitions, Phys. Rev. B, 1978, 17(3), 1302-1322 DOI: 10.1103\%2Fphysrevb.17.1302

[35] van Gunsteren, W.; Berendsen, H.: Algorithms for brownian dynamics, Mol. Phys., 1982, 45(3), 637647 DOI: $10.1080 \% 2 F 00268978200100491$

[36] Brünger, A.; Brooks, C.L.; Karplus, M.: Stochastic boundary conditions for molecular dynamics simulations of ST2 water, Chem. Phys. Lett., 1984, 105(5), 495-500 DOI: 10.1016\%2F00092614\%2884\%2980098-6

[37] Leimkuhler, B.; Matthews, C.: Rational Construction of Stochastic Numerical Methods for Molecular Sampling, Appl. Math. Res. eXpress, 2013, 2013(1), 34-56 DOI: 10.1093\%2Famrx\%2Fabs010

[38] Horváth, B.; Szalai, I.: Structure of electrorheological fluids: A dielectric study of chain formation, Phys. Rev. E, 2012, 86(6),061403 DOI: $10.1103 \% 2$ Fphysreve.86.061403

[39] Horváth, B.; Szalai, I.: Dynamic dielectric response of electrorheological fluids in drag flow, Phys. Rev. E, 2015, 92(4), 042308 DOI: 10.1103\%2Fphysreve.92.042308

[40] Berti, C.; Furini, S.; Gillespie, D.; Boda, D.; Eisenberg, R.S.; Sangiorgi, E.; Fiegna, C.: A 3D Brownian Dynamics simulator for the study of ion permeation through membrane pores, $J$. Chem. Theor. Comput., 2014, 10(8), 2911-2926 DOI: $10.1021 / \mathrm{ct} 4011008$ 